\title{
Whence metal-free clouds?
}

Mon. Not. R. Astron. Soc. (in the press); preprint

available at https://arxiv.org/abs/1812.05098

An intergalactic cloud at a redshift of $z \approx 4.4$ is composed of nothing other than hydrogen and helium, report Frédéric Robert and collaborators. High-resolution spectra obtained towards a background quasar show no hints of metal absorption lines, implying that the cloud has a metallicity of less than one ten-thousandth of the Sun's. The origin of the cloud is unclear, but it may result from the collected ejecta of low-energy, lowmass population III supernovae, or it may have fortunately remained in a condition unsullied by stellar debris since the Big Bang.

The cloud under study (LLS1723) is an example of a Lyman limit system (LLS), meaning that it has a sufficient column density of neutral hydrogen to make the cloud optically thick bluewards of the Lyman limit at $912 \AA$. Three other highredshift LLSs are known, at lower redshifts just beyond $z=3$, and two of these similarly do not exhibit any metal lines. Keck HIRES spectra of LLS1723 over $\sim 4,800-9,200 \AA$ reveal many hydrogen lines (that constrain the column density to $\log _{10}\left(N_{\mathrm{H}_{\mathrm{I}}} / \mathrm{cm}^{-2}\right)=$ 17.9-18.3), but strong transitions of $\mathrm{O}$, C IV, Si II and Si Iv are absent, and weak $\mathrm{C}_{\text {II }}$ features at $1,334 \AA$ (rest frame) are likely to be telluric.

There are two main origin scenarios for the very metal-poor LLSs: they are pristine, cold streams of accreting gas (as seen in cosmological simulations) that are present in the circumgalactic medium, or they populate the intergalactic medium. A simple discriminant would be to map the Ly $\alpha$ emitting environment around these LLSs using optical integral field spectroscopy to look for nearby galaxies.

\section{Paul Woods}

Published online: 31 January 2019 https://doi.org/10.1038/s41550-019-0707-1 